\title{
The Effect of a Self Exercise Program in Cardiac Rehabilitation for Patients with Coronary Artery Disease
}

\author{
Chul Kim, M.D., Jo Eun Youn, M.D., Hee Eun Choi, M.D.
}

Department of Rehabilitation Medicine, Sanggye Paik Hospital, Inje University College of Medicine, Seoul 139-707, Korea

Objective To investigate the effect of self exercise in cardiac rehabilitation on cardiopulmonary exercise capacity for selected patients with coronary artery disease.

Method The subjects of this study were patients who received percutaneous coronary intervention (PCI) or coronary artery bypass graft (CABG) surgery and who participated in a cardiac rehabilitation program. The supervised exercise group participated in 6-8 weeks of aerobic exercise training with telemetry ECG monitoring in hospital. The self exercise group, whose exercise risk was low, was instructed to participiate in self exercise training in a community exercise environment according to the exercise tolerance test (ETT) using a modified Bruce protocol. Both groups underwent ETTs before and 6 months after initiation of the cardiac rehabilitation program. We compared the supervised group with the self exercise groups on exercise capacity.

Results After 6 months, the supervised exercise group showed significant changes in maximum oxygen consumption, maximal heart rate, resting heart rate, and submaximal rate pressure product. The self exercise group also showed significant improvement of maximum oxygen consumption and submaximal rate pressure product. However, the changing rate of maximum oxygen consumption was significantly higher in the supervised exercise group than the self exercise group.

Conclusion Both the supervised and self exercise groups showed similar improvement of cardiopulmonary exercise capacity after 6 months' participation in the cardiac rehabilitation program. However, the changing rate of maximum oxygen consumption, maximal heart rate, and resting heart rate were significantly higher in the supervised exercise group than the self exercise group.

Key Words Coronary artery disease, Exercise, Rehabilitation, Self

Received August 9, 2010; Accepted November 19, 2010

Corresponding author: Hee Eun Choi

Department of Rehabilitation Medicine, Sanggye Paik Hospital, Inje University College of Medicine 761-1, Sanggye 7 dong, Nowon-gu, Seoul 139-707, Korea

Tel: +82-2-950-1134, Fax: +82-2-935-3076, E-mail: che0070@hanmail.net Copyright $\odot 2011$ by Korean Academy of Rehabilitation Medicine

\section{INTRODUCTION}

It is known that cardiac rehabilitation programs can improve patients' quality of life and exercise capacity and reduce the rate of heart attack recurrence and mortality. ${ }^{1-5}$ Cardiac rehabilitation programs traditionally consist of three phases: inpatient, outpatient, and community maintenance. ${ }^{6}$ Outpatient programs are carefully 
performed under the supervision of health care providers with monitoring based on exercise tolerance test results. Despite the numerous benefits of cardiac rehabilitation programs, the participation rates still remain very low, ${ }^{7}$ and it has been suggested that this is due to patients' difficulties and problems with medical services. ${ }^{8}$ Several obstacles that can impede patient participation have been reported, including occupational and domestic issues, a lack of interest in rehabilitation, a reluctance to make lifestyle changes, depression, or a lack of family cooperation. ${ }^{9-13}$ In our previous study, it was found that one of the foremost barriers to participation was the lack of awareness of rehabilitation programs $(78 \%)$. Other barriers included the patients' doubt of positive effects, a lack of time, fear, high cost, poor physical condition, and poor accessibility to program centers due to distance and traffic problems. The lack of awareness and the patients' regional or socioeconomic conditions were particularly responsible for rehabilitation discontinuation. ${ }^{14}$

Community-based self-exercise programs can be primarily applicable to patients with a low risk of heart attack if they sufficiently understand the significance of cardiac rehabilitation, but they have difficulty accessing the programs due to their regional and socioeconomic problems. It is expected that self exercise programs will be beneficial for patients who cannot attend supervised exercise programs in hospitals. For successful performance, the program should be designed to improve the exercise capacity of patients with coronary disease as supervised programs do, and the program should include only patients with a low rate of cardiovascular events. After demonstrating efficacy and safety, self exercise programs can be used for a wide range of patients, and these programs can improve their cardiopulmonary function. Therefore, these programs will be more cost-effective and have better effects on reducing the recurrence of heart attack and the mortality rate. We aimed to compare the results of self-exercise programs and supervised programs for 6 to 8 weeks after screening and selecting low-risk patients.

\section{MATERIALS AND METHODS}

\section{Study subjectives}

We enrolled patients who were admitted to our cardiovascular center with acute coronary syndrome (ACS) between January 2007 and December 2008, or those who were referred to the cardiac rehabilitation clinic after undergoing percutaneous coronary intervention (PCI) or a coronary artery bypass graft (CABG). The exclusion criteria included prior PCI or CABG; left ventricular ejection fraction (LVEF) less than $40 \%$, uncontrolled arrhythmia, hypertension, or diabetes; as well as additional issues that would preclude a potential patient from participation in the program.

All the eligible patients were recommended to participate in cardiac rehabilitation programs by means of educating them about the benefits and contents of the programs. The patients who notified us of their intention to attend were assigned into the supervised exercise group. Those who were not able to present in the hospital were assigned into the self exercise group if they were categorized as "low-risk" according to the Risk Classification for Exercise Training, as established by the American Association of Cardiovascular and Pulmonary Rehabilitation (AACVPR) (Table 1). ${ }^{15}$

We followed 91 patients; 45 patients were assigned into the supervised exercise group and 46 were assigned into the self exercise group. The supervised exercise group was composed of 38 men (84\%) and 7 women (16\%), and the self exercise group was composed of 38 men $(83 \%)$ and 8

Table 1. Risk Classification for Exercise Training by AACVPR Guidelines

\begin{tabular}{lcc}
\hline $\begin{array}{l}\text { Variables for high risk at } \\
\text { initial ETT }\end{array}$ & $\begin{array}{c}\text { Supervised } \\
\text { exercise } \\
(\mathbf{n}=\mathbf{4 5})\end{array}$ & $\begin{array}{c}\text { Self } \\
\text { exercise } \\
(\mathbf{n}=\mathbf{4 6})\end{array}$ \\
\hline $\begin{array}{l}\text { Sustained VT or SVT } \\
\begin{array}{l}\text { Functional capacity } \\
\text { (METs < } 6)\end{array}\end{array}$ & 7 & - \\
$\begin{array}{l}\text { Drop in SBP }>15 \mathrm{mmHg} / \\
\text { failure to rise with exercise } \\
\text { /any drop in SBP with } \\
\text { exercise }\end{array}$ & $3 / 2$ & - \\
ST Depression $>2$ mm & - & - \\
$\begin{array}{l}\text { Angina (+) } \\
\text { LVEF < 30\% }\end{array}$ & - & - \\
\hline $\begin{array}{l}\text { AACVPR: American Association of Cardiovascular and } \\
\text { Pulmonary Rehabilitation, ETT: Exercise tolerance } \\
\text { test, VT: Ventricular tachycardia, SVT: Supra venticular }\end{array}$ \\
tachycardia, MET: Metabolic equivalent of the task, SBP: \\
$\begin{array}{l}\text { Systolic blood pressure, LVEF: Left ventricular ejection } \\
\text { fraction }\end{array}$
\end{tabular}


Table 2. Baseline Characteristics of Subjects

\begin{tabular}{|c|c|c|c|}
\hline & $\begin{array}{l}\text { Supervised } \\
\text { exercise } \\
(n=45)\end{array}$ & $\begin{array}{c}\text { Self } \\
\text { exercise } \\
(n=46)\end{array}$ & p-value \\
\hline Number of subjects & 45 & 46 & $>0.05$ \\
\hline Sex (male : female) & $38: 7$ & $38: 8$ & $>0.05$ \\
\hline Age (years) & 57.6 & 57.4 & $>0.05$ \\
\hline Procedure : PCI & 27 & 29 & $>0.05$ \\
\hline CABG & 18 & 17 & $>0.05$ \\
\hline $\begin{array}{l}\text { Number of involved } \\
\text { vessel }\end{array}$ & 2.5 & 2.2 & $>0.05$ \\
\hline $\operatorname{LVEF}(\%)$ & 60.5 & 61.4 & $>0.05$ \\
\hline $\mathrm{VO}_{2 \max }(\mathrm{ml} / \mathrm{kg} / \mathrm{min})$ & 26.2 & 28.6 & $>0.05$ \\
\hline
\end{tabular}

PCI: Percutaneous coronary intervention, CABG: Coronary artery bypass graft, LVEF: Left ventricular ejection fraction, $\mathrm{VO}_{2 \max }$ : Maximal oxygen consumption

women (17\%). There was no significant difference in the gender distribution between the two groups. The mean age was 57.6 in the supervised group and 57.4 in the self group; the mean age was not significantly different. In addition, there was no significant difference in the rate of patients who underwent PCI or CABG, the number of affected coronary arteries, the LVEF at baseline, and the maximal oxygen consumption $\left(\mathrm{VO}_{2 \max }\right)$ in both groups (Table 2).

\section{Method}

Echocardiographic studies were performed within 48 hours before and after PCI in all the patients, and symptom-limited exercise tolerance testing was carried out using the modified Bruce protocol within a week after discharge from the hospital. A real-time recording 12-channel ECG (Q4500, Quinton Instrument Co., Boston, USA), a respiratory gas analyzer (QMC, Quinton Instrument CO., Boston, USA), an automatic blood pressure and pulse monitor (Model 412, Quinton Instrument Co., Boston, USA), and a treadmill (Medtrack ST 55, Quinton Instrument Co., Boston, USA) were included in the test. The $\mathrm{VO}_{2 \max }$ was measured with a respiratory gas analyzer and the maximum heart rate, the stable heart rate, and the myocardial oxygen demand $\left(\mathrm{MVO}_{2}\right)$ were estimated by the ECG and the automatic blood pressure and pulse monitor. The $\mathrm{MVO}_{2}$ was calculated by multiplying the systolic blood pressure and heart rate as the rate pressure product (RPP). The submaximal $\mathrm{MVO}_{2}$ was measured at the end of stage 3 of the modified Bruce protocol.

The patients in the supervised group were monitored with an ECG based on the results of exercise tolerance testing during the program in the hospital. A Quinton MEDTRACK SR60 Treadmill (Quinton Instrument Co., Seattle, USA) and a Quinton CORIVAL 400 ergometer bicycle (Quinton Instrument Co., Seattle, USA) were used in the program. The exercise intensity was increased in a stepwise manner based on the target heart rate. The target heart rate was supposed to be $40 \%$ to $85 \%$ of the value of the heart rate reserve, which can be calculated with the maximum heart rate and stable heart rate obtained from exercise tolerance testing. The supervised exercise programs were mostly of a 6- to 8-week duration, three sessions per week with a total of 50 minutes per session. Each exercise session was divided into a 10-minute warm-up, 30 minutes of prescribed exercise, and a 10-minute cool-down. After completing the program, we advised the patients to participate in self exercise programs based on their target heart rate and the rate of perceived exertion obtained from re-tested exercise tolerance. The patients in the self group were prescribed exercise based on the results of the exercise tolerance testing performed within a week after discharge, and they were advised to participate in community-based self exercise programs for 6 months without a period of supervised training. The prescribed exercise included fast walking, power walking, bicycling, and jogging, depending upon the individual's exercise capacity and systemic conditions.

Information on risk factors and therapy was given to all the patients and their families. They were also counseled for smoking cessation, obesity, and nutrition, and we monitored their adherence to exercise and risk factor management with blood tests, body fat, and blood pressure measurements, in addition to exercise counseling every 1 to 3 months. Symptom limited exercise tolerance was re-tested in both groups using the modified Bruce protocol after an initial 6-month period of the programs. The extent of improvement and the pattern of cardiopulmonary capacity were compared between the two groups at baseline and 6 months. Severe cardiovascular complications during the study period were also investigated. 


\section{Statistical analyses}

The data was statistically analyzed with SAS Enterprise Guide 4.1 (4.1.0.471). We used Chi-square tests to compare the gender distribution and the rate of PCI and CABG, and the Wilcoxon rank sum test was used to compare ages, the LVEF, the $\mathrm{VO}_{2 \max }$, the maximum heart rate, the stable heart rate, and the maximal and submaximal $\mathrm{MVO}_{2}$ at baseline and 6 months. The Wilcoxon signed rank test was used to compare the $\mathrm{VO}_{2 \max }$, the maximum heart rate, the stable heart rate, and the maximal and submaximal (Phase III exercise) RPPs in each group at baseline and 6 months. A p-value $<$ 0.05 was considered statistically significant.

\section{RESULTS}

Severe cardiovascular complications during exercise

According to the AACVPR, 18 of 45 patients had highrisk factors on the risk classification for exercise training in the supervised group, whereas none of the 46 patients in the self exercise group had high-risk factors (Table 1). Severe cardiovascular complications did not occur in either group during the study.

Comparing the amount of cardiopulmonary capacity change

In the supervised group, the $\mathrm{VO}_{2 \max }$ and maximum heart rate increased while the stable heart rate and submaximal $\mathrm{MVO}_{2}$ decreased significantly compared to baseline. Similarly, the $\mathrm{VO}_{2 \max }$ increased and the submaximal $\mathrm{MVO}_{2}$ decreased in the self exercise group, but no significant change in either the maximum heart rate or stable heart rate was observed. The maximal $\mathrm{MVO}_{2}$ tended to decrease in the supervised group, and it increased in the self group, but these changes were not statistically significant (Table 3 ).

Comparing the rate of change of cardiopulmonary capacity

There was no significant difference in the rate of maximum heart rate change, the stable heart rate, and the maximal and submaximal $\mathrm{MVO}_{2}$ between the two groups. The rate of change of the $\mathrm{VO}_{2 \max }$ increased more significantly in the supervised group than that seen in the self group (21\% vs. $8.6 \%, \mathrm{p}<0.05)$ (Table 4$)$.
Table 3. Comparison of Parameters between Supervised and Self Exercise

\begin{tabular}{lrrrr}
\hline & \multicolumn{2}{c}{$\begin{array}{c}\text { Supervised } \\
\text { exercise }(\mathbf{n}=45)\end{array}$} & \multicolumn{2}{c}{$\begin{array}{c}\text { Self exercise } \\
(\mathbf{n}=46)\end{array}$} \\
\cline { 2 - 5 } & Before & After & Before & After \\
\hline $\mathrm{HR}_{\text {max }}$ (beats $\left./ \mathrm{min}\right)$ & 138.5 & $147.4^{*}$ & 139.2 & 140.5 \\
$\mathrm{HR}_{\text {rest }}($ beats $/ \mathrm{min})$ & 78.8 & $71.6^{*}$ & 73.8 & 71.2 \\
$\mathrm{RPP}_{\text {max }}(\mathrm{mmHg} \times \mathrm{bpm})$ & 27,420 & 26,905 & 24,633 & 24,685 \\
$\mathrm{RPP}_{\text {submax }}(\mathrm{mmHg} \times \mathrm{bpm})$ & 17,144 & $14,609^{*}$ & 15,373 & $13,745^{*}$ \\
$\mathrm{VO}_{2 \text { max }}(\mathrm{ml} / \mathrm{kg} / \mathrm{min})$ & 26.2 & $30.7^{*}$ & 28.6 & $30.7^{*}$ \\
\hline
\end{tabular}

$\mathrm{HR}_{\text {max }}$ : Maximal heart rate, $\mathrm{HR}_{\text {rest }}$ : Resting heart rate, $\mathrm{RPP}_{\max }$ : Maximal rate pressure product, $\mathrm{RPP}_{\text {submax }}$ : Submaximal rate pressure product at stage $3, \mathrm{VO}_{2 \max }$ : Maximal oxygen consumption ${ }^{*} \mathrm{p}<0.05$ before vs. after

Table 4. Changing Rate of Parameters between Supervised and Self Exercise

\begin{tabular}{lcc}
\hline & $\begin{array}{c}\text { Supervised exercise } \\
(\mathbf{n}=45)\end{array}$ & $\begin{array}{c}\text { Self exercise } \\
(\mathbf{n}=\mathbf{4 6})\end{array}$ \\
\hline $\mathrm{HR}_{\text {max }}(\%)$ & +7.3 & +1.5 \\
$\mathrm{HR}_{\text {rest }}(\%)$ & -8.4 & -1.7 \\
$\mathrm{RPP}_{\text {max }}(\%)$ & -11.6 & +2.8 \\
$\mathrm{RPP}_{\text {submax }}(\%)$ & -10.5 & -5.4 \\
$\mathrm{VO}_{2 \max }(\%)$ & $+21.1^{*}$ & +8.6 \\
\hline
\end{tabular}

$\mathrm{HR}_{\text {max }}$ : Maximal heart rate, $\mathrm{HR}_{\text {rest }}$ : Resting heart rate, $\mathrm{RPP}_{\max }$ : Maximal rate pressure product, $\mathrm{RPP}_{\text {submax }}$ : Submaximal rate pressure product at stage $3, \mathrm{VO}_{2 \max }$ : Maximal oxygen consumption

${ }^{*} \mathrm{p}<0.05$ supervised exercise vs. self exercise, ${ }^{\dagger}$ Changing rate $=(B-A) / A \times 100$; A: Baseline, B: Results after 6 months

\section{DISCUSSION}

Coronary artery disease has a high prevalence and high mortality rates all over the world. Due to advances in medical technology, the mortality rate seems to have diminished, but the prevalence rate has gradually increased ${ }^{16-18}$ It has been demonstrated that cardiac rehabilitation programs, such as those that include exercise programs, nutrition counseling, smoking cessation programs, pharmaceutical therapy, and weight control, play a key role in improving exercise capacity and secondary prevention for patients with coronary artery disease. ${ }^{19,20}$ In a meta-analysis of 48 randomized 
clinical trials, it was found that cardiac rehabilitation programs reduced the all-cause mortality by $20 \%$ and the cardiovascular mortality by $27 \% .^{21,22}$ Despite these benefits, cardiac rehabilitation programs have been underused, and the rate of discontinuation is very high. Many reasons for the low participation rates have been reported, such as poor access to hospitals ${ }^{23-25}$ and the lack of time due to occupational or household work. ${ }^{26-29} \mathrm{~A}$ self exercise program was first introduced at the beginning of the 1980s. It can be an alternative to traditional programs, and it has been extensively used for patients due to its easy accessibility and convenience. ${ }^{30}$

Exercise training is a critical component of cardiac rehabilitation programs. Aerobic exercise can reduce the myocardial burden when performing daily physical activities because aerobic exercise increases the $\mathrm{VO}_{2 \max }$ and it decreases the submaximal heart rate. ${ }^{31-33}$ In addition, the $\mathrm{VO}_{2}$ in the systemic muscles may be reduced, and as these muscles are needed to exercise with the same intensity, reduction in the $\mathrm{VO}_{2}$ lessens the myocardial burden in patients with heart disease. The incidence of myocardial ischemia can be reduced by decreasing the $\mathrm{MVO}_{2}$ because myocardial ischemia generally occurs at the same RPP. We suggest that improvement of cardiopulmonary capacity was achieved in both groups because the $\mathrm{VO}_{2 \max }$ increased and the submaximal $\mathrm{MVO}_{2}$ decreased. Consequently, this relieved the patients' cardiopulmonary symptoms, and enhanced functional capacity may be expected in daily and socio-occupational activities.

Several foreign reports that have published the results of comparative studies on the improvement of exercise capacity with supervised and self exercise programs, the management of risk factors, and the quality of life. According to the recent Cochrane meta-analysis, no difference was observed in cardiopulmonary exercise capacity, risk factors management, health-related quality of life, and recurrence of heart disease (death, reperfusion, and readmission) between supervised and self exercise programs during short term and long term follow-up. ${ }^{34}$ Jolly et al. ${ }^{30}$ reported that exercise capacity, systolic blood pressure, and total cholesterol were improved more in the supervised group, but no statistically significant difference was seen. In our study, the $\mathrm{VO}_{2 \max }$ significantly increased and the $\mathrm{MVO}_{2}$ significantly decreased both in the supervised group and the self group; however, statistically significant increases of the maximal heart rate and significant decreases of the stable heart rate were found only in the supervised group. Specifically, the change rate of the $\mathrm{VO}_{2 \max }$ was greater in the supervised group $(\mathrm{p}<0.05)$. One possible explanation for this is that the exercise compliance was much greater in the supervised group than in that of the self group even though the patients were closely monitored and guided. Furthermore, it is already known that the lower the functional capacity is, as measured before initiating cardiac rehabilitation programs, the greater the improvement may be after completion of the programs.

However, safety and legal parameters concerning prevention of heart attack are not currently established; therefore, self exercise programs should be carefully used. Patients should always be tested to determine whether they are eligible for the programs by evaluating the risk of cardiac attack with exercise tolerance testing. Severe cardiovascular complications associated with exercise did not occur in any of our patients. A possible explanation is that the 18 patients with a high risk of heart attack were assigned into supervised programs, whereas no high-risk patients were assigned to the self exercise group. Moreover, the patients in the supervised group had their exercise intensity controlled in real time by close monitoring at the beginning of each exercise session.

There are some limitations in our study. First, this is not a randomized comparative study. The patients decided to participate in cardiac rehabilitation programs by themselves, and the patients at a high risk of heart attack during exercise were excluded from the self exercise group. Second, we did not clearly determine differences in the management scores of several risk factors in both groups, including hypertension, diabetes mellitus, hyperlipidemia, obesity, and smoking cessation. Finally, long-term follow-up after 6 months was not done. Additional studies may be necessary to compare the improvement in cardiopulmonary functional capacity with long-term exercise as well as the recurrence of heart attack and mortality rates and cost-effectiveness.

\section{CONCLUSION}

We performed 6-month cardiac rehabilitation programs for patients with prior PCI or CABG due to coronary 
artery disease. We demonstrated that cardiopulmonary exercise capacity significantly improved in both groups. However, the change of the $\mathrm{VO}_{2 \max }$ maximum heart rate, and stable heart rate improved more in the supervised exercise group than in the self exercise group. Further large-scale, long-term follow-up studies will be required in the future.

\section{REFERENCES}

1. Taylor RS, Brown A, Ebrahim S, Jolliffe J, Noorani H, Rees K, Skidmore B, Stone JA, Thompson DR, Oldridge N. Exercise-based rehabilitation for patients with coronary heart disease: systematic review and meta-analysis of randomized controlled trials. Am J Med 2004; 116: 682-692

2. O'Connor GT, Buring JE, Yusuf S, Goldhaber SZ, Olmstead EM, Paffenbarger RS Jr. Hennekens CH. An overview of randomized trials of rehabilitation with exercise after myocardial infarction. Circulation 1989; 80: $234-244$

3. Dugmore LD, Tipson RJ, Phillips MH, Flint EJ, Stentiford NH, Bone MF, Littler WA. Changes in cardiopulmonary fitness, psychological wellbeing, quality of life, and vocational status following a 12 month cardiac exercise rehabilitation programme. Heart 1999; 81: 359-366

4. Izawa K, Hirano Y, Yamada S, Oka K, Omiya K, Iijima S. Improvement in physiological outcomes and healthrelated quality of life following cardiac rehabilitation in patients with acute myocardial infarction. Circulation 2004; 68: 315-320

5. Witt BJ, Jacobsen SJ, Weston SA, Killian JM, Meverden RA, Allison TG, Reeder GS, Roger VL. Cardiac rehabilitation after myocardial infarction in the community. J Am Coll Cardiol 2004; 44: 988-996

6. Whiteson JH. Cardiac rehabilitation. In: Braddom RL, editor. Physical medicine and rehabilitation, 3rd ed, Philadelphia: Sauders, 2007, 723

7. Bunker S, McBurney H, Cox H, Jelinek M. Identifying participation rates at outpatient cardiac rehabilitation programs in Victoria, Australia. J Cardiopulm Rehabil 1999; 19: 334-338

8. Beswick AD, Rees K, Griebsch I, Taylor FC, Burke M, West RR, Victory J, Brown J, Taylor RS, Ebrahim S. Provision, uptake and cost of cardiac rehabilitation programmes: improving services to under-represented groups. Health Technol Assess 2004; 8(41): 1152

9. Pell J, Pell A, Morrison C, Blatchford O, Dargie H. Retrospective study of influence of deprivation on uptake of cardiac rehabilitation. BMJ 1996; 313: 267268

10. Evenson KR, Fleury J. Barriers to outpatient cardiac rehabilitation participation and adherence. J Cardiopulm Rehabil 2000; 20: 241-246

11. Lane D, Carroll D, Ring C, Beevers DG, Lip GY. Predictors of attendance at cardiac rehabilitation after myocardial infarction. J Psychosom Res 2001; 51: 497 501

12. Blumenthal JA, Williams RS, Wallace AG, Williams RB Jr, Needles TL. Physiological and psychological variables predict compliance to prescribed exercise therapy in patients recovering from myocardial infarction. Psychosom Med 1982; 44: 519-527

13. Gulanick M, Bliley A, Perino B, Keough V. Recovery patterns and lifestyle changes after coronary angioplasty: the patient's perspective. Heart Lung 1998; 27: 253-262

14. Kim C, Lim HS, Ahn JK, Bang IK, Lee SM, Kim YJ. The reasons that cardiac patients did not participate in and drop out from the cardiac rehabilitation program. J Korean Acad Rehab Med 2002; 26: 790-796

15. The American Association of Cardiovascular and Pulmonary Rehabilitation. Guidelines for cardiac rehabilitation and secondary prevention programs. Human kinetics 1999; 3: 45

16. Neal B. Secondary prevention of cardiovascular disease: fixed dose combinations. In: Kaplan W, Laing R, editors. Priority medicines for Europe and the world. World Health Organization, Geneva, Switzerland, 2004

17. Law MR, Watt HC, Wald NJ. The underlying risk of death after myocardial infarction in the absence of treatment. Arch Intern Med 2002; 162: 2405-2410

18. World Health Organization. The World Health Report 1997- conquering suffering, enriching humanity. World Health Forum 1997; 18: 248-260

19. Ades PA. Cardiac rehabilitation and secondary prevention of coronary heart disease. New Engl J Med 2001; 345: 892-902

20. Balady GJ, Ades PA, Comoss P, Limacher M, Pina 
IL, Southard D, Williams MA, Bazzarre T. Core components of cardiac rehabilitation/secondary prevention programs: a statement for healthcare professionals from the American Heart Association and the American Association of Cardiovascular and Pulmonary Rehabilitation Writing Group. Circulation 2000; 102: 1069-1073

21. Jolliffe JA, Rees K, Taylor RS, Thompson D, Oldridge N, Ebrahim S. Exercise-based rehabilitation for coronary heart disease. Cochrane Database Syst Rev 2001; 1: CD001800

22. Taylor RS, Brown A, Ebrahim S, Jolliffe J, Noorani H, Rees K, Skidmore B, Stone JA, Thompson DR, Oldridge N. Exercise-based rehabilitation for patients with coronary heart disease: systematic review and meta-analysis of randomized controlled trials. Am J Med 2004; 116: 682-692

23. Ades PA. Cardiac rehabilitation and secondary prevention of coronary heart disease N Engl J Med 2001; 345: 892-902

24. Ades PA, Waldmann ML, McCann WJ, Weaver SO. Predictors of cardiac rehabilitation participation in older coronary patients. Arch Intern Med 1992; 152: 1033-1035

25. Andrew GM, Oldridge NB, Parker JO, Cunningham DA, Rechnitzer PA, Jones NL, Buck C, Kavanagh T, Shephard RJ, Sutton JR. Reasons for dropout from exercise programs in post coronary patients. Med Sci Sports Exerc 1981; 13: 164-168

26. Pell J, Pell A, Morrison C, Blatchford O, Dargie H. Retrospective study of influence of deprivation on uptake of cardiac rehabilitation. BMJ 1996; 313: 267268

27. Nohara R, Kambara H, Hohiuddin IH, Ono S, Okuda
K, Makita S, Hamazaki H, Aoto K, Shimomura M, Hayashi M, et al. Cardiac sports rehabilitation for patients with ischemic heart disease. Jpn Circ J 1990; 54: 1443-1450

28. Evenson K, Fleury J. Barriers to outpatient cardiac rehabilitation participation and adherence. J Cardiopulm Rehabil 2000; 20: 241-246

29. Cannistra LB, Balady GJ, O'Malley CJ, Weinger DA, Ryan TJ. Comparison of the clinical profile and outcome of women and men in cardiac rehabilitation. Am J Cardiol 1992; 69: 1274-1279

30. Jolly K, Taylor RS, Lip GY, Stevens A. Home-based cardiac rehabilitation compared with centre-based rehabilitation and usual care: a systematic review and meta-analysis. Int J Cardiol 2006; 111: 343-351

31. Kim C, Lim SW, Lee SM, Ahn JK. Effectiveness of aerobic exercise in cardiac patients. J Korean Acad Rehab Med 2000; 24: 1155-1160

32. Kraus WE, Houmard JA, Duscha BD, Knetzger KJ, WhartonMB, McCartney JS, Bales CW, Henes S, Samsa GP, Otvos JD, et al. Effects of the amount and intensity of exercise on plasma lipoproteins. N Eng J Med 2002; 347: 1483-1492

33. Fletcher GF, Balady GJ, Amsterdam EA, Chaitman B, Eckel R, Fleg J, Froelicher VF, Leon AS, Pina IL, Rodney R, et al. Exercise standards for testing and training: a statement for healthcare professionals from the American Heart Association. Circulation 2001; 104: 1694- 1740

34. Dalal HM, Zawada A, Jolly K, Moxham T, Taylor RS. Home based versus centre based cardiac rehabilitation: cochrane systematic review and metaanalysis. BMJ 2010; 340: b5631 